\title{
Umbral Calculus and \\ Cancellative Semigroup Algebras
}

\author{
V. V. Kisil \\ Dedicated to the memory of Gian-Carlo Rota
}

\begin{abstract}
We describe some connections between three different fields: combinatorics (umbral calculus), functional analysis (linear functionals and operators) and harmonic analysis (convolutions on group-like structures). Systematic usage of cancellative semigroups, their convolution algebras, and tokens between them provides a common language for description of objects from these three fields.

Keywords: Cancellative semigroups, umbral calculus, harmonic analysis, tokens, convolution algebras, integral transforms
\end{abstract}

AMS subject classification: 43 A 20, 05 A 40, $20 \mathrm{~N}++$

\section{Introduction}

We discuss some interactions between the representation theory of semigroups, umbral calculus in combinatorics, and linear operators in functional analysis. Our main bridge between them is the notion of cancellative semigroups, convolution algebras over them and tokens from such a semigroup to another.

We look for a vocabulary, which is reasonably general and allows us to translate without alterations as much about umbral calculus as possible. The language we select relies on convolution algebras. Such algebras arise from essentially different sources like groups, posets, or linear operators. The goal of the present paper is to study properties of convolutions algebras, which are independent from their origins.

Umbral calculus was known long ago but it was not well understood till very recent time. During its history it took many different faces: linear functionals [14], Hopf algebras [13], axiomatic definition [19] to list only few different interpretations.

The goal of our paper is to give a model, which realizes described features by means of common objects. What is the purpose of such models? One can think on the Poincaré model of non-Euclidean geometry in a disk. The model does not only demonstrate the

V. V. Kisil: Dept. Pure Math. of the Univ., Leeds LS2 9JT, UK; kisilv@amsta.leeds.ac.uk Supported by grant 3GP03196 of the FWO-Vlaanderen (Fund of Scientific Research-Flanders), Scientific Research Network "Fundamental Methods and Technique in Mathematics". 
logical consistency of Lobachevski's geometry. Another (no less important) psychological function is to express the unusual geometry via objects of the intuitive classic geometry. The logical consistence of umbral calculus is evident (from papers [13, 14, 19], for example). But it is our feeling that some more links with fundamental objects are still desirable.

The paper presents basic definitions and properties of cancellative semigroups, tokens, convolution algebras, and their relations with umbral calculus. We will illustrate our consideration mostly by the simplest example: the cancellative semigroup of nonnegative integers $\mathbb{N}_{+}$. More non-trivial examples will be present elsewhere.

The layout is as follows. In Section 2 we sketch three different interpretations of umbral calculus from papers $[13,14,19]$. Section 3 introduces cancellative semigroups, convolutions, and tokens and their basic properties. We use them in Section 4 to describe principal combinatorial objects like delta families, generating functions, and recurrence operators. We conclude the paper with Section 5 which links our construction with three realizations of umbral calculus from $[13,14,19]$ recalled in Section 2.

\section{Umbral calculus}

Umbral calculus was put on a solid ground by G.-C. Rota and collaborates [11, 13, 14, 19 ] in three different ways at least. We repeat here some essential definitions from these papers.

2.1 Finite operator description. The three ways are based essentially on the two following fundamental notions [16]:

1. A polynomial sequence of binomial type, that is, a sequence of polynomials $p_{n} \quad\left(\operatorname{deg} p_{n}=n\right)$ with complex coefficients, satisfying the identities

$$
p_{n}(x+y)=\sum_{k=0}^{n} p_{k}(x) p_{n-k}(y)
$$

Our definition is different from the original one - we take polynomials divided by $n$ !. Our choice will be explained shortly in connection with the group property. Such form of the defining identity together with the more accurate name polynomial sequence of integral type were used in [4]. Paper [10] also start from this formula, the polynomials are called there convolution polynomials and their investigation is made with the help of Mathematica software.

2. A shift-invariant operator, namely a linear operator $S$ on the vector space $\mathcal{P}$ of all such polynomials, which commutes with the ordinary derivative $D p=p^{\prime}$, that is, an operator $S$ with the property $T D p=D T p$ for all polynomials $p$. Within shift invariant operators the following one plays an exceptional role: an operator $Q$ is said to be a delta operator (associated to a polynomial sequence $p_{n}$ ) when

$$
Q p_{n}=p_{n-1} \quad \text { or, more general, } \quad Q^{k} p_{n}=p_{n-k}
$$


2.2 Hopf algebras description. There is a canonical isomorphism between shiftinvariant operators and linear functionals (see Proposition 3.11), thus we can think on powers of delta $Q^{k}$ as functionals $q_{k}$ on polynomials connected with an associated polynomial sequence $p_{n}$ by duality:

$$
\left\langle q_{k}, p_{n}\right\rangle=\delta_{k n}
$$

which easily follows from (2.2). Here $\delta_{k n}$ is the Kronecker delta ${ }^{1)}$. We are going to show that delta operators and polynomial sequences are not only dual notions, but two faces of the same object - tokens between cancellative semigroups (Examples 3.15 and $3.16)$.

2.3 The semantic description. This subsection contains definitions from [18, 19].

Definition 2.1 (see [18]). An umbral calculus is an ordered quadruple, consisting of the following items:

1. A commutative integral domain $D$ whose quotient field is of characteristic zero.

2. An alphabet $A$ whose elements are termed umbrae and denoted by Greek letters. This alphabet generates the polynomial ring $D[A]$, the elements of which are termed umbral polynomials.

3. A linear functional eval: $D[A] \rightarrow D$, such that eval $(1)=1$, i.e. eval leaves $D$ fixed, and such that

$$
\operatorname{eval}\left(\alpha^{i} \beta^{j} \cdots \gamma^{k}\right)=\operatorname{eval}\left(\alpha^{i}\right) \operatorname{eval}\left(\beta^{j}\right) \cdots \operatorname{eval}\left(\gamma^{k}\right)
$$

where $\alpha, \beta, \ldots, \gamma$ are distinct umbrae.

4. A distinguished umbrae $\varepsilon$ in $A$, such that eval $\left(\varepsilon^{i}\right)=\delta_{i, 0}$. The umbrae $\varepsilon$ is sometimes called the augmentation.

Definition 2.2 (see [18]). Let $f$ and $g$ be two umbral polynomials in $D[A]$. Then $f$ and $g$ are said to be umbrally equivalent, written $f \simeq g$, when eval $(f)=\operatorname{eval}(g)$.

Definition 2.3 (see [18]). Two umbral polynomials $p$ and $q$ are termed exchangeable, written $p \equiv q$, when $p^{n} \simeq q^{n}$ for all $n \geq 0$.

1) We use the same letter $\delta$ to denote also the Dirac delta function. The Kronecker delta $\delta_{k n}$ and the Dirac function $\delta(x)$ can be distinguished by their arguments. 


\section{Cancellative semigroups and tokens}

3.1 Cancellative semigroups: definition and examples. Our consideration is based on the following notion of semigroups with cancellation or cancellative semigroups [3: Section IV.1.1].

Definition 3.1. A semigroup $C$ is called a left or a right cancellative semigroup if for any $a, b, c \in C$ the identity $c a=c b$ or $a c=b c$, respectively, implies $a=b$. A cancellative semigroup is both a left and a right cancellative semigroup.

Equivalently, a left or right cancellative semigroup is defined by the condition that for arbitrary $a, b \in C$ the equation $a \cdot x=b$ or $x \cdot a=b$, respectively, has at most one solution (if any). We hope that the readability of the paper will be better if we use the notion c-semigroup to denote a cancellative semigroup. We also use the notion of a $c$-set $C$ (which is weaker than c-semigroup) by allowing the multiplication $(a, b) \mapsto a b$ on $C$ be defined only on a proper subset of $C \times C$.

Definition 3.2. An element $e$ is called a (left, right) source of a (left, right) csemigroup $C$ if both equations (the first equation, the second equation) $x \cdot e=b$, $e \cdot x=b$ do (does, does) have a (unique) solution for any $b \in C$.

Obviously, a (left, right) identity on $C$ will be a (left, right) source.

We still denote the unique-if-exist solutions to equations $x a=b$ and $a x=b$ by $\left[b a^{-1}\right]$ and $\left[a^{-1} b\right]$, respectively. Here the braces stress that both $\left[b a^{-1}\right]$ and $\left[a^{-1} b\right]$ are monosymbols and just " $a^{-1}$ " is not defined in general. Let us also assume that all c-semigroups under consideration can be equipped by an invariant measure $d b$, namely $d b=d(a b)$ for all $a \in C$. We will not discuss herein conditions for its existence or possible modifications of our constructions for the case of quasi-invariant measures.

C-semigroups were investigated as an algebraic object in connection with groups. It is particularly known that any commutative c-semigroup can be embedded in a group, but this is not necessary true for a non-commutative c-semigroup [3: Section IV.1.1]. Our motivation in this object is the following. If we have a group $G$, then the important associated object is the right regular (linear) representation by "shifts" $\pi_{g} f(h)=f(h g)$ in $\mathcal{L}_{p}(G, d \mu)$. Then one can introduce their linear span - convolutions:

$$
K f(h)=\int_{G} k(g) \pi_{g} d g f(h)=\int_{G} k(g) f(h g) d g .
$$

Thus one may consider the composition of convolutions $K_{2} K_{1}$ with two kernels $k_{1}, k_{2} \in$ $\mathcal{L}_{1}(G)$ :

$$
\begin{aligned}
K_{2} K_{1} f(h) & =\int_{G} k_{2}\left(g_{2}\right) \int_{G} k_{1}\left(g_{1}\right) f\left(h g_{2} g_{1}\right) d g_{1} d g_{2} \\
& =\int_{G}\left(\int_{G} k_{2}\left(g_{2}\right) k_{1}\left(g_{2}^{-1} g\right) d g_{2}\right) f(h g) d g
\end{aligned}
$$

where $g=g_{2} g_{1}$. Thereafter it again is a convolution with the kernel

$$
k(g)=\int_{G} k_{2}\left(g_{2}\right) k_{1}\left(g_{2}^{-1} g\right) d g_{2} .
$$


An important role in the consideration is played by the unique solvability of equation $g_{1} g_{2}=g$ with respect to $g_{1}$. For an algebraic structure weaker than a group, if the inverse $g^{-1}$ is not defined, one can try to define a composition of convolutions by the formula

$$
\left(k_{1} * k_{2}\right)(h)=\iint_{g_{1} g_{2}=h} k_{1}\left(g_{1}\right) k_{2}\left(g_{2}\right) d g_{1} d g_{2} .
$$

However, such a definition generates problems with the understanding of the set double integral (3.3) is taken over and the corresponding measure. These difficulties disappear for left c-semigroups: for given $b$ and $a$ there is at most one such $x=\left[a^{-1} b\right]$ that $a x=b$. Thus we can preserve for a c-semigroup $C$ definition (3.2) with the small modification

$$
\left(k_{2} * k_{1}\right)(b)=\int_{C} k_{2}(a) k_{1}\left(\left[a^{-1} b\right]\right) d a .
$$

To avoid problems with the definition of convolution we make the agreement that for any function $f$

$$
f\left(\left[a^{-1} b\right]\right)=0 \quad \text { when }\left[a^{-1} b\right] \text { does not exist in } C
$$

(i.e., the equation $a x=b$ does not have a solution). Our agreement is equivalent to the introduction of an incidence algebra of functions $f(a, b)$, such that $f(a, b)=0$ for $a>b$ for a poset (see Example 3.7).

Remark 3.3. As soon as one passes directly to convolution algebras and abandon points of c-semigroups themselves, future generalizations in the spirit of noncommutative geometry [6] are possible.

We give several examples of c-semigroups now.

Example 3.4 (Principal). The main source of c-semigroups is the following construction. Let us have a set $S$ with an additional structure $\mathcal{A}$. Then the set of all mappings from $S$ into $S$, which preserve $\mathcal{A}$, forms a right c-semigroup.

Example 3.5. If one considers in Example 3.4 only mappings $S$ onto $S$, then they give us a definition of groups. Moreover, a large set of c-semigroups can be constructed from groups directly. Namely, let $C$ be a subset of a group $G$ such that $C \cdot C \subset C$. Then $C$ with multiplication induced from $G$ will be a c-semigroup. In such a way we obtain the c-semigroup of positive real (natural, rational) numbers with the usual addition. Positive entire numbers with multiplication also form a c-semigroup. If $C$ contains the identity $e$ of $G$, then $e$ is a source of $C$. Particularly, entire group $G$ is also a c-semigroup. We should note that even for groups some of our technique will be new (for example tokens), at least up to the author knowledge.

The last example demonstrates that we have many c-semigroups with (left, right) invariant measures such that Fubini's theorem holds. This follows from corresponding constructions for groups.

We sign out the most important for the present paper example from the described family and his alternative realization. 
Proposition 3.6. The algebra of formal power series in a variable $t$ is topologically isomorphic to the convolution algebra $\mathcal{C}\left(\mathbb{N}_{+}\right)$of continuous functions with point-wise convergence over the c-semigroup of non-negative integers $\mathbb{N}_{+}$with the discrete topology. The subalgebra of polynomials in one variable $t$ is isomorphic to the convolution algebra $\mathcal{C}_{0}\left(\mathbb{N}_{+}\right)$, which is dense in $\mathcal{C}\left(\mathbb{N}_{+}\right)$, of compactly supported functions on $\mathbb{N}_{+}$.

The proof is hardly needed. Note that "puzzling questions" about convergence of formal power series and continuity of functionals on them correspond to the natural topology on $\mathcal{C}\left(\mathbb{N}_{+}\right)$.

A less obvious family of examples can be constructed from a structure quite remoted (at least at the first glance) from groups.

Example 3.7. Let $P$ be a poset (i.e. partialy ordered set) and let $C$ denote the subset of the Cartesian square $P \times P$ such that $(a, b) \in C$ if and only if $a \leq b(a, b \in P)$. We can define a multiplication on $C$ by the formula

$$
(a, b)(c, d)= \begin{cases}\text { undefined } & \text { if } b \neq c \\ (a, d) & \text { if } b=c .\end{cases}
$$

One can see that $C$ is a c-set. If $P$ is locally finite, i.e. for any $a \leq b \quad(a, b \in P)$ the number of $z$ between $a$ and $b$ (i.e. $a \leq z \leq b$ ) is finite, then we can define a measure $d(a, b)=1$ on $C$ for any $(a, b) \in C$. With such a measure (3.4) defines a correct convolution on $C$ :

$$
h(a, b)=\int_{C} f(c, d) g\left(\left[(c, d)^{-1}(a, b)\right]\right) d(c, d)=\sum_{a \leq z \leq b} f(a, z) g(z, b) .
$$

The constructed algebra is the fundamental incidence algebra in combinatorics [7].

Our present technique can be successfully applied only to c-semigroups, not to csets. Thus it is an important observation that the reduced incidence algebra construction [7: Section 4] contracts c-sets to c-semigroups in many important cases (however, not in general). Particularly, all combinatorial reduced incidence algebras listed in [7: Examples $4.5-4.9]$ are convolution algebras over c-semigroups. However, the incidence coefficients should be better understood.

Example 3.8. Any groupoid (see [22] and references herein) is a c-set.

After the examples given, it is reasonable to expect an applicability of c-semigroups to combinatorics. We describe applications in the next section explicitly.

Now we point out some basic properties of c-semigroups, trying to be as near as possible to their prototype - groups. All results will be stated for left c-semigroups. Under agreement (3.5) we have

Lemma 3.9 (Shift invariance of integrals). For any function $f$ we have

$$
\int_{C} f(c) d c=\int_{C} f\left(\left[a^{-1} b\right]\right) d b \quad \forall a \in C .
$$

Proof. It follows from the observation that the integrals in both sides of (3.8) are taken over the whole $C$ and that the measure $d b$ is invariant 
One should be warned that $\int_{C} f(c) d c \neq \int_{C} f(a b) d b$ for c-semigroups (unlike for groups) in general as well as Lemma 3.9 is false for c-sets. Another very useful property (which c-semigroups possess from groups) is a connection between linear functionals and shift invariant operators. Again unlike in the group case we should make the right choice of shifts.

Definition 3.10. We define a left shift operator $\lambda_{a}$ on the space of function $\mathcal{L}_{1}(C)$ by the formula $\left[\lambda_{a} f\right](b)=f(a b)$. A linear operator $S$ is (left) shift-invariant if $S \lambda_{a}=$ $\lambda_{a} S$ for all $a \in C$. The augmentation $\varepsilon$ associated to a right source $e$ is the linear functional defined by $\varepsilon(f)=f(e)$.

Proposition 3.11. Let $C$ be a c-semigroup with a right source $e$. The following three spaces are in one-to-one correspondence:

1. The space $\mathcal{B}(L)$ of liner functionals on the space of functions $\mathcal{L}(C)$.

2. The space $\mathcal{B}(S)$ of shift invariant operators.

3. The algebra of convolutions $\mathfrak{C}$ with functions from $\mathcal{L}^{*}(C)$.

The correspondences are given by the formulas $\left(l \in \mathcal{L}^{*}(C), S \in \mathcal{S}, k \in \mathfrak{C}, f \in \mathcal{L}(C)\right)$

$$
\begin{array}{ll}
l \rightarrow S & \text { where }[S f](a)=\left\langle l, \lambda_{a} f\right\rangle \\
S \rightarrow l & \text { where } l f=\varepsilon(S f) \\
k \rightarrow l & \text { where } l f=\int_{C} k(c) f(c) d c \\
k \rightarrow S & \text { where } S f(a)=\int_{C} k(c) f(a c) d c .
\end{array}
$$

Proof. Let us have a linear shift invariant operator $S$. Clearly, its composition $l f=\varepsilon S f=[S f](e)$ with the augmentation is a linear functional. By the definition of distributions [9: Section III.4] such a linear functional can be represented as integral $l k=\int_{C} k(c) f(c) d c$ with a (probably generalized) function $k$ from the dual space. For any function $f$ we have $f(a)=\lambda_{\left[a e^{-1}\right]} f(e)$, thus by the shift-invariance of $S, S f(a)=$ $\left[\lambda_{\left[a e^{-1}\right]} S f\right](e)=\left[S \lambda_{\left[a e^{-1}\right]} f\right](e)$ or, by the definition of $l, S f(a)=\left\langle l, \lambda_{\left[a e^{-1}\right]} f\right\rangle$. The integral form of the last identity is $S f(a)=\int_{C} k(c) f(a c) d c$, which coincides with (3.1)

An essential rôle in all three approaches (operators, Hopf algebras, semantics) to umbral calculus is played by linear functionals (and thus associated linear shift-invariant operators). Connections between linear functionals and shifts can be greatly simplified if we are able to express shifts via some "linear coefficients" of functions. The known tool to do that is the Fourier transform. An alternative tool for c-semigroups is presented in the next section.

3.2 Tokens: Definition and Examples. We formalize identity (2.1) in the following notion, which will be useful in our consideration and seems to be of interest for the representation theory. The main usage of this notion is t-transform defined in the next subsection.

Definition 3.12. Let $C_{1}$ and $C_{2}$ be two c-semigroups. We will say that a function $t\left(c_{1}, c_{2}\right)$ on $C_{1} \times C_{2}$ is a token from $C_{1}$ to $C_{2}$ if for any $c_{1}^{\prime} \in C_{1}$ and any $c_{2}, c_{2}^{\prime} \in C_{2}$ we have

$$
\int_{C_{1}} t\left(c_{1}, c_{2}\right) t\left(\left[c_{1}^{-1} c_{1}^{\prime}\right], c_{2}^{\prime}\right) d c_{1}=t\left(c_{1}^{\prime}, c_{2} c_{2}^{\prime}\right)
$$


Remark 3.13. We derive this definition "experimentally" from (2.1) (see also the examples bellow). However, one can discover it "theoretically" as well. We already know from Proposition 3.11 that a shift-invariant operator $S$ can be represented as a combination of a shift and a linear functional: $[S f](a)=\left\langle l, \lambda_{a} f\right\rangle$. This suggests to represent a function $f$ as a linear combination

$$
f(b)=\int e(b, c) \hat{f}(c) d c
$$

of elementary components $e(b, c)$, which behave simply under shifts. Here "simply" means do not destroy linear combination (3.10).

A way to achieve this is provided by the Fourier transform. Here $e(b, c)=e^{i b c}$ and the action of a shift $\lambda_{a}$ reduces to multiplication:

$$
\lambda_{a} e(b, c)=e(b+a, c)=e^{i a c} e(b, c) .
$$

This probably is the simplest solution but not the only possible one. More general transformation of this sort is given by abstract wavelets (or coherent states):

$$
\lambda_{a} e(b, c)=e(b+a, c)=\int k\left(a, c, c_{1}\right) e\left(b, c_{1}\right) d c_{1}
$$

where we can particularly select $k\left(a, c, c_{1}\right)=e^{i a c} \delta\left(c-c_{1}\right)$ to get the rule of exponents (3.11) and $k\left(a, c, c_{1}\right)=e\left(c_{1}^{-1} c, a\right)$ to get the token property (3.9). Then shifts act on functions defined via (3.10) as

$$
\begin{aligned}
\lambda_{a} f(b) & =\lambda_{a} \int e(b, c) \hat{f}(c) d c \\
& =\iint k\left(a, c, c_{1}\right) e\left(b, c_{1}\right) d c_{1} \hat{f}(c) d c \\
& =\int e\left(b, c_{1}\right)\left(\int k\left(a, c, c_{1}\right) \hat{f}(c) d c\right) d c_{1} .
\end{aligned}
$$

Thus it again looks like (3.10) where the action of a shift reduced to an integral operator on the symbol $\hat{f}(c)$. In the case of token shifts affect in the way (3.9), which stand between the extreme simplicity of (3.11) and the almost unaccessible generality of (3.12). This analogy with the Fourier transform will be employed in the next subsection for the definition of t-transform.

Remark 3.14. Relationships between harmonic analysis (group characters) and token-like structures (polynomial of binomial type) was already pointed in [5]. Note that tokens are a complementary (in some sense) tool for the exponent $e^{i b c}$. While both are particularly useful in the investigation of shift invariant operators they work in different ways. The Fourier transform maps shift-invariant operators to operators of multiplication. t-transform defined in the next subsection by means of tokens maps shift-invariant operators on one c-semigroup to shift-invariant operators on another csemigroup.

We present examples of tokens within classical objects. 
Example 3.15. Let $C_{1}$ be the c-semigroup of non-negative natural numbers $\mathbb{N}_{+}$ and $C_{2}$ be the c-semigroup (a group in fact) of real numbers, both equipped by addition. Let $t(n, x)=p_{n}(x)$, where $p_{n}$ is a polynomial sequence of binomial type. Then the characteristic property of a sequence (2.1) is equal to the definition of token (3.9) read from the right to the left.

Example 3.16. We exchange $C_{1}$ and $C_{2}$ from the previous example, they are real numbers and non-negative natural numbers, respectively. Let us take a shiftinvariant operator $L$. Any its power $L^{n}$ is again a shift-invariant operator represented by a convolution with a function $l(n, x)$ with respect to the variable $x$. The identity $L^{n+k}=L^{n} L^{k}$ can be expressed in the term of the correspondent kernels of convolutions:

$$
\int_{\mathbb{R}} l(n, x) l(k, y-x) d x=l(n+k, y) .
$$

The first two examples with their explicit duality generate a hope that our development can be of some interest for combinatorics. But before working out this direction we would like to present some examples of tokens outside combinatorics.

Example 3.17. Let $C_{1}=\mathbb{R}^{n}$ and $C_{2}=\mathbb{R}^{n} \times \mathbb{R}_{+}-$the "upper half space" in $\mathbb{R}^{n+1}$. For the space of harmonic functions in $C_{2}$ there is an integral representation over the boundary $C_{1}$ :

$$
f(v, t)=\int_{C_{1}} P(u ; v, t) f(u) d u \quad\left(u \in C_{1},(v, t) \in C_{2}\right) .
$$

Here $P$ is the celebrated Poisson kernel $P(u ; v, t)=\frac{2}{\left|S_{n}\right|} \frac{t}{\left(|u-v|^{2}+t^{2}\right)^{\frac{n+1}{2}}}$ with the property usually referred as a semigroup property [2: Chapter 3/Prob. 1]

$$
P\left(u ; v+v^{\prime}, t+t^{\prime}\right)=\int_{C_{1}} P\left(u^{\prime} ; v^{\prime}, t^{\prime}\right) P\left(u-u^{\prime} ; v, t\right) d u^{\prime} .
$$

We meet the token in analysis.

Example 3.18. We preserve the meaning of $C_{1}=\mathbb{R}^{n}$ and $C_{2}=\mathbb{R}^{n} \times \mathbb{R}_{+}$from the previous example and define the Weierstrass (or Gauss-Weierstrass) kernel by the formula

$$
W(z ; w, \tau)=\frac{1}{(\sqrt{2 \pi \tau})^{n}} e^{-\frac{|z-w|^{2}}{2 \tau}}=\frac{1}{(2 \pi)^{n}} \int_{\mathbb{R}^{n}} e^{-\frac{\tau}{2}|u|^{2}} e^{-(u, z-w)} d u
$$

where $z \in C_{1}$ and $(w, \tau) \in C_{2}$. The function $W$ is the fundamental solution to the heat equation [8: Section 2.3]. We again have [2: Chapter3, Prob. 1]

$$
W\left(z ; w+w^{\prime}, \tau+\tau^{\prime}\right)=\int_{C_{1}} W\left(z^{\prime} ; w^{\prime}, \tau^{\prime}\right) W\left(z-z^{\prime} ; w, \tau\right) d z
$$

Thus we again meet a token.

Examples 3.17 and 3.18 open a huge list of integral kernels [2] which are tokens of analysis. We will see the role of tokens for analytic function theory and reproducing kernels later.

We finish the subsection by the following elementary property of tokens. 
Lemma 3.19. Let the c-semigroup $C_{2}$ has a left source $e_{2}$. Then $k\left(c_{1}\right)=t\left(c_{1}, e_{2}\right)$ has the reproducing property

$$
t\left(c_{1}^{\prime}, c_{2}\right)=\int_{C_{1}} k\left(c_{1}\right) t\left(\left[c_{1}^{-1} c_{1}^{\prime}\right],\left[e_{2}^{-1} c_{2}\right]\right) d c_{1}
$$

for all $c_{1}^{\prime} \in C_{1}$ and $c_{2} \in C_{2}$. Particularly, if $e_{2} \in C_{2}$ is a left unit, then

$$
t\left(c_{1}^{\prime}, c_{2}\right)=\int_{C_{1}} k\left(c_{1}\right) t\left(\left[c_{1}^{-1} c_{1}^{\prime}\right], c_{2}\right) d c_{1}
$$

for all $c_{1}^{\prime} \in C_{1}$ and $c_{2} \in C_{2}$.

Proof. We apply the characteristic property of a token (3.9) for particularly selected elements in $C$ :

$$
\begin{aligned}
t\left(c_{1}^{\prime}, c_{2}\right) & =t\left(c_{1}^{\prime}, e_{2}\left[e_{2}^{-1} c_{2}\right]\right) \\
& =\int_{C_{1}} t\left(c_{1}, e_{2}\right) t\left(\left[c_{1}^{-1} c_{1}^{\prime}\right],\left[e_{2}^{-1} c_{2}\right]\right) d c_{1} \\
& =\int_{C_{1}} k\left(c_{1}\right) t\left(\left[c_{1}^{-1} c_{1}^{\prime}\right],\left[e_{2}^{-1} c_{2}\right]\right) d c_{1}
\end{aligned}
$$

and the statement is proved

3.3 t-transform. We would like now to introduce a transformation associated with tokens (t-transform). t-transform is similar to the Fourier and wavelet transforms. The main difference - we do not insist that our transformation maps convolutions to multiplications. We will be much more modest - our transformation maps convolutions to other convolutions, eventually more simple or appropriate.

Having a function $\hat{f}\left(c_{1}\right)$ on $C_{1}$ we can consider it as coefficients (or a symbol) and define the function $f\left(c_{2}\right)$ on $C_{2}$ by means of the token $t\left(c_{1}, c_{2}\right)$ :

$$
f\left(c_{2}\right)=\int_{C_{1}} \hat{f}\left(c_{1}\right) t\left(c_{1}, c_{2}\right) d c_{1}
$$

We will say that $f\left(c_{2}\right) \in \mathcal{A}\left(C_{2}\right)$ if in (3.14) $\hat{f}\left(c_{1}\right) \in \mathcal{C}_{0}\left(C_{1}\right)$ - the space of continuous functions on $C_{1}$ with compact support. So we can consider the token $t\left(c_{1}, c_{2}\right)$ as a kernel of an integral transform $\mathcal{C}_{0}\left(C_{1}\right) \rightarrow \mathcal{A}\left(C_{2}\right)$. The use of the notation reserved for the Fourier transform ("hat") is justified (at least partially) by Remark 3.13.

We would like to mention the following

Corollary 3.20. The reproducing property of $k\left(c_{1}\right)$ from Lemma 3.19 can be extended by linearity to $\mathcal{A}\left(C_{1}\right)$ :

$$
f\left(c_{1}^{\prime}\right)=\int_{C_{1}} k\left(c_{1}\right) f\left(\left[c_{1}^{-1} c_{1}^{\prime}\right]\right) d c_{1} .
$$


Example 3.21. We continue with a polynomial sequence of binomial type $p_{k}(x)$, which was presented as a token $p(k, x)$ in Example 3.15. Having few numbers $\hat{a}_{1}, \hat{a}_{2}, \ldots$, which are interpreted as a function $\hat{a}(k)$ in $\mathcal{C}_{0}\left(\mathbb{N}_{+}\right)$, we can construct a polynomial

$$
a(x)=\int_{\mathbb{N}_{+}} \hat{a}(k) p(k, x) d k=\sum_{k} \hat{a}_{k} p_{k}(x),
$$

that is a function on $\mathbb{R}$.

Example 3.22. Let $\delta^{(k)}$ be a token from $\mathbb{R}$ to $\mathbb{N}_{+}$(Example 3.16). Then for a smooth at point 0 function $f$ the integral transformation $f^{(k)}(0)=\int_{\mathbb{R}} f(x) \delta^{(k)}(x) d x$ produces a function on $\mathbb{N}_{+}$.

Example 3.23. Let a real-valued function $f$ be defined on $C_{1}=\mathbb{R}^{n}$. Then we can define the function $P f$ on $C_{2}=\mathbb{R}^{n} \times \mathbb{R}_{+}$by means of the Poisson integral:

$$
P f(v, t)=\int_{C_{1}} P(u ; v, t) f(u) d u=\frac{2}{\left|S_{n}\right|} \int_{\mathbb{R}^{n}} \frac{t}{\left(|u-v|^{2}+t^{2}\right)^{\frac{n+1}{2}}} f(u) d u .
$$

The image is a harmonic function solving the Dirichlet problem (see [20: Appendix/Section 2] and [8: Subsection 2.2.4]).

Example 3.24. Similarly we define the Weierstrass transform

$$
W f(w, \tau)=\int_{C_{1}} W(z ; w, \tau) f(z) d z=\frac{1}{(\sqrt{2 \pi \tau})^{n}} \int_{\mathbb{R}^{n}} e^{-\frac{|z-w|^{2}}{2 \tau}} f(z) d z .
$$

The image is a function satisfying the heat equation.

Let $K$ be a convolution on $C_{2}$ with kernel $k$. Then it acts on a function $f$ defined by $(3.14)$ as

$$
\begin{aligned}
{[K f]\left(c_{2}\right) } & =\int_{C_{2}} k\left(c_{2}^{\prime}\right) f\left(c_{2} c_{2}^{\prime}\right) d c_{2}^{\prime} \\
& =\int_{C_{2}} k\left(c_{2}^{\prime}\right) \int_{C_{1}} \hat{f}\left(c_{1}^{\prime}\right) t\left(c_{1}^{\prime}, c_{2} c_{2}^{\prime}\right) d c_{1}^{\prime} d c_{2}^{\prime} \\
& =\int_{C_{2}} k\left(c_{2}^{\prime}\right) \int_{C_{1}} \hat{f}\left(c_{1}^{\prime}\right) \int_{C_{1}} t\left(c_{1}, c_{2}\right) t\left(\left[c_{1}^{-1} c_{1}^{\prime}\right], c_{2}^{\prime}\right) d c_{1} d c_{1}^{\prime} d c_{2}^{\prime} \\
& =\int_{C_{1}} \int_{C_{1}} \int_{C_{2}} k\left(c_{2}^{\prime}\right) t\left(\left[c_{1}^{-1} c_{1}^{\prime}\right], c_{2}^{\prime}\right) d c_{2}^{\prime} \hat{f}\left(c_{1}^{\prime}\right) d c_{1}^{\prime} t\left(c_{1}, c_{2}\right) d c_{1} \\
& =\int_{C_{1}} \int_{C_{1}} \int_{C_{2}} k\left(c_{2}^{\prime}\right) t\left(c_{1}^{\prime \prime}, c_{2}^{\prime}\right) d c_{2}^{\prime} \hat{f}\left(c_{1} c_{1}^{\prime \prime}\right) d c_{1}^{\prime \prime} t\left(c_{1}, c_{2}\right) d c_{1} \\
& =\int_{C_{1}}\left(\int_{C_{1}}[\mathcal{T} k]\left(c_{1}^{\prime \prime}\right) \hat{f}\left(c_{1} c_{1}^{\prime \prime}\right) d c_{1}^{\prime \prime}\right) t\left(c_{1}, c_{2}\right) d c_{1}
\end{aligned}
$$

where

$$
[\mathcal{T} k]\left(c_{1}^{\prime \prime}\right)=\int_{C_{2}} k\left(c_{2}^{\prime}\right) t\left(c_{1}^{\prime \prime}, c_{2}^{\prime}\right) d c_{2}^{\prime} .
$$

We used in (3.15) the characteristic property of tokens (3.9) and the change of variables $c_{1}^{\prime \prime}=\left[c_{1}^{-1} c_{1}^{\prime}\right]$ in (3.16). Note that big brasses in (3.17) contain a convolution on $C_{1}$ with kernel (3.18). So transformation (3.14) maps convolutions on $C_{2}$ to convolutions on $C_{1}$.

The mapping deserves a special name. 
Definition 3.25. t-transform is a linear integral transformation $\mathcal{C}\left(C_{2}\right) \rightarrow \mathcal{C}\left(C_{1}\right)$ defined on kernels by the formula

$$
[\mathcal{T} k]\left(c_{1}\right)=\int_{C_{2}} t\left(c_{1}, c_{2}\right) k\left(c_{2}\right) d c_{2}
$$

According to Lemma 3.9 we have

$$
[\mathcal{T} k]\left(c_{1}\right)=\int_{C_{2}} t\left(c_{1},\left[c_{2}^{-1} c_{2}^{\prime}\right]\right) k\left(\left[c_{2}^{-1} c_{2}^{\prime}\right]\right) d c_{2}^{\prime} \quad \forall c_{2} \in C_{2}
$$

The t-transform is more than just a linear map:

Theorem 3.26. $t$-transform is an algebra homomorphism of the convolution algebra $\mathfrak{C}\left(C_{2}\right)$ to the convolution algebra $\mathfrak{C}\left(C_{1}\right)$, namely

$$
\left[\mathcal{T}\left(k_{1} * k_{2}\right)\right]=\left[\mathcal{T} k_{1}\right] *\left[\mathcal{T} k_{2}\right]
$$

where $*$ in the left-hand side denotes the composition of convolutions on $C_{2}$ and in the right-hand side on $C_{1}$.

Proof. We silently assume that the conditions of Fubini's theorem are fulfilled. It turns to be that Fubini's theorem will be our main tool, which is used in almost all proofs. Thereafter we assume that it fulfils through the entire paper. We have:

$$
\begin{aligned}
{[\mathcal{T}} & \left.\left(k_{2} * k_{1}\right)\right]\left(c_{1}^{\prime}\right) \\
& =\int_{C_{2}} t\left(c_{1}^{\prime}, c_{2}^{\prime}\right) \int_{C_{2}} k_{2}\left(c_{2}\right) k_{1}\left(\left[c_{2}^{-1} c_{2}^{\prime}\right]\right) d c_{2} d c_{2}^{\prime} \\
& =\int_{C_{2}} \int_{C_{2}} \int_{C_{1}} t\left(c_{1}, c_{2}\right) t\left(\left[c_{1}^{-1} c_{1}^{\prime}\right],\left[c_{2}^{-1} c_{2}^{\prime}\right]\right) d c_{1} k_{2}\left(c_{2}\right) k_{1}\left(\left[c_{2}^{-1} c_{2}^{\prime}\right]\right) d c_{2} d c_{2}^{\prime} \\
& =\int_{C_{1}} \int_{C_{2}} t\left(c_{1}, c_{2}\right) k_{2}\left(c_{2}\right) d c_{2} \int_{C_{2}} t\left(\left[c_{1}^{-1} c_{1}^{\prime}\right],\left[c_{2}^{-1} c_{2}^{\prime}\right]\right) k_{1}\left(\left[c_{2}^{-1} c_{2}^{\prime}\right]\right) d c_{2}^{\prime} d c_{1} \\
& =\int_{C_{1}}\left[\mathcal{T} k_{2}\right]\left(c_{1}\right)\left[\mathcal{T} k_{1}\right]\left(\left[c_{1}^{-1} c_{1}^{\prime}\right]\right) d c_{1} .
\end{aligned}
$$

Of course, we use in transformation (3.22) the characteristic property of the tokens (3.9) and conclusion (3.23) is based on (3.20)

Knowing the connection between shift invariant operators and convolutions (Proposition 3.11) we derive

Corollary 3.27. $t$-transform maps left shift-invariant operators on $C_{2}$ to left shiftinvariant operators on $C_{1}$. 


\section{Shift invariant operators}

In this section we present some results in terms of c-semigroups, tokens and t-transforms and illustrate them by examples from combinatorics and analysis.

4.1 Delta families and basic distributions. This subsection follows the paper [17] with appropriate modifications.

Definition 4.1. Let $C_{1}$ and $C_{2}$ be two c-semigroups, and let $e_{1}$ be a right source of $C_{1}$. We will say that linear shift-invariant operators $Q\left(c_{1}\right) \quad\left(c_{1} \in C_{1}\right)$ on $\mathcal{A}\left(C_{2}\right)$ over a c-semigroup $C_{2}$ form a delta family if

1. for a token $t\left(c_{1}, c_{2}\right), Q\left(c_{1}\right) t\left(c_{1}, c_{2}\right)=t\left(e_{1}, c_{2}\right)$ for all $c_{2} \in C_{2}$

2. the semigroup property $Q\left(c_{1}\right) Q\left(c_{1}^{\prime}\right)=Q\left(c_{1} c_{1}^{\prime}\right)$ hold.

Example 4.2. Let $C_{1}$ and $C_{2}$ be as in Examples 3.15 and 3.21. Let also $t(n, x)=$ $\frac{x^{n}}{n !}$. Then the family of positive integer powers of the derivative $Q(n)=D^{n}$ forms a delta family. Other delta families for given $C_{1}$ and $C_{2}$ are listed in [12: Section 3]. They are positive integer powers of difference, backward difference, central difference, Laguerre, and Abel operators.

Example 4.3. For the Poisson kernel from Example 3.17 we can introduce a delta family as Euclidean shift operators $Q(x) f(v, t)=f(v+x, t) \quad\left(x \in \mathbb{R}^{n}\right)$.

Proposition 4.4. Let $q\left(c_{1}, c_{2}\right)$ be the kernel corresponding to a delta family $Q\left(c_{1}\right)$ as a convolution over $C_{2}$. Then $q\left(c_{1}, c_{2}\right)$ is a token from $C_{2}$ to $C_{1}$. Namely,

$$
q\left(c_{1} c_{1}^{\prime}, c_{2}^{\prime}\right)=\int_{C_{2}} q\left(c_{1}, c_{2}\right) q\left(c_{1}^{\prime},\left[c_{2}^{-1} c_{2}^{\prime}\right]\right) d c_{2} .
$$

Proof. This is a simple restatement in terms of kernels of the semigroup property $Q\left(c_{1}\right) Q\left(c_{1}^{\prime}\right)=Q\left(c_{1} c_{1}^{\prime}\right)$

Definition 4.5. A function $t\left(c_{1}, c_{2}\right)$ is called the basic distribution for a delta family $Q\left(c_{1}\right)$ if

1. $t\left(c_{1}, e_{2}\right)=k\left(c_{1}\right)$ has the reproducing property from Lemma 3.19 over $C_{1}$ (particularly, if $\left.k\left(c_{1}\right)=\delta\left(c_{1}\right)\right)$

2. $Q\left(c_{1}\right) t\left(c_{1}^{\prime}, c_{2}\right)=t\left(\left[c_{1}^{-1} c_{1}^{\prime}\right], c_{2}\right)$.

Example 4.6. For the classic umbral calculus this conditions turn to be $p_{0}(x)=1$, $p_{n}(0)=0(n>0)$ and $Q p_{n}=p_{n-1}$.

Example 4.7. For the Poisson integral the reproducing property can be obtained if we consider a generalized Hardy space $\mathcal{H}_{2}\left(\mathbb{R}_{+}^{n+1}\right)$ of $\mathcal{L}_{2}$-integrable functions on $\mathbb{R}^{n}$, which are limit value of harmonic functions in the upper half space $\mathbb{R}^{n} \times \mathbb{R}_{+}$. Then the kernel $k\left(u-u^{\prime}\right)$ is defined as a distribution given by the limit of integral on $\mathcal{H}_{2}\left(\mathbb{R}^{n}\right)$ :

$$
\begin{aligned}
f(u) & =\int_{\mathbb{R}^{n}} k\left(u-u^{\prime}\right) f\left(u^{\prime}\right) d u^{\prime} \\
& =\frac{2}{\left|S_{n}\right|} \lim _{(v, t) \rightarrow(0,0)} \int_{\mathbb{R}^{n}} \frac{t}{\left(\left|\left(u-u^{\prime}\right)-v\right|^{2}+t^{2}\right)^{\frac{n+1}{2}}} f\left(u^{\prime}\right) d u^{\prime} .
\end{aligned}
$$


This follows from the boundary property of the Poisson integral (see [20: Appendix/Section 2] and [8: Subsection 2.2.4]).

Theorem 4.8. Let $C_{1}$ and $C_{2}$ be c-semigroups.

1. If $t\left(c_{1}, c_{2}\right)$ is a basic distribution for some delta family $Q\left(c_{1}\right) \quad\left(c_{1} \in C_{1}\right)$, then $t\left(c_{1}, c_{2}\right)$ is a token from $C_{1}$ to $C_{2}$.

2. If $t\left(c_{1}, c_{2}\right)$ is a token from $C_{1}$ to $C_{2}$, then it is a basic distribution for some delta family $Q\left(c_{1}\right) \quad\left(c_{1} \in C_{1}\right)$.

Proof. Let $t\left(c_{1}, c_{2}\right)$ be a basic distribution for $Q\left(c_{1}\right)$ and let $q\left(c_{2}, c_{1}\right)$ be a kernel of the operator $Q\left(c_{1}\right)$ as a convolution on $C_{2}$. This means that

$$
\left\langle q\left(c_{2}, c_{1}\right), t\left(c_{1}^{\prime}, c_{2}\right)\right\rangle=k\left(\left[c_{1}^{-1} c_{1}^{\prime}\right]\right)
$$

has a reproducing property due to Lemma 3.19 and Corollary 3.20. Thus we can trivially express $t\left(c_{1}^{\prime}, c_{2}^{\prime}\right)$ as integral $t\left(c_{1}^{\prime}, c_{2}^{\prime}\right)=\int_{C_{1}} t\left(c_{1}, c_{2}^{\prime}\right)\left\langle q\left(c_{2}, c_{1}\right), t\left(c_{1}^{\prime}, c_{2}\right)\right\rangle d c_{1}$. By linearity we have a similar expression $f\left(c_{2}^{\prime}\right)=\int_{C_{1}} t\left(c_{1}, c_{2}^{\prime}\right)\left\langle q\left(c_{2}, c_{1}\right), f\left(c_{2}\right)\right\rangle d c_{1}$ for any function in $\mathcal{A}\left(C_{2}\right)$. In particular,

$$
t\left(c_{1}^{\prime}, c_{2}^{\prime \prime} c_{2}^{\prime}\right)=\int_{C_{1}} t\left(c_{1}, c_{2}^{\prime}\right)\left\langle q\left(c_{2}, c_{1}\right), t\left(c_{1}^{\prime}, c_{2}^{\prime \prime} c_{2}\right)\right\rangle d c_{1} .
$$

But

$$
\begin{aligned}
\left\langle q\left(c_{2}, c_{1}\right), t\left(c_{1}^{\prime}, c_{2}^{\prime \prime} c_{2}\right)\right\rangle & =\left.Q\left(c_{1}\right) \lambda_{c_{1}^{\prime \prime}} t\left(c_{1}^{\prime}, c_{2}\right)\right|_{c_{2}=e_{2}} \\
& =\left.\lambda_{c_{2}^{\prime \prime}} Q\left(c_{1}\right) t\left(c_{1}^{\prime}, c_{2}\right)\right|_{c_{2}=e_{2}} \\
& =\left.\lambda_{c_{2}^{\prime \prime}} t\left(\left[c_{1}^{-1} c_{1}^{\prime}\right], c_{2}\right)\right|_{c_{2}=e_{2}} \\
& =t\left(\left[c_{1}^{-1} c_{1}^{\prime}\right], c_{2}^{\prime \prime}\right)
\end{aligned}
$$

and therefore

$$
t\left(c_{1}^{\prime}, c_{2}^{\prime \prime} c_{2}^{\prime}\right)=\int_{C_{1}} t\left(c_{1}, c_{2}^{\prime}\right) t\left(\left[c_{1}^{-1} c_{1}^{\prime}\right], c_{2}^{\prime \prime}\right) d c_{1},
$$

that is, the distribution $t\left(c_{1}, c_{2}\right)$ is a token.

Suppose now that $t\left(c_{1}, c_{2}\right)$ is a token. We define a family of operators $Q\left(c_{1}\right)$ on $\mathcal{A}\left(C_{2}\right)$ by identities $Q\left(c_{1}\right) t\left(c_{1}^{\prime}, c_{2}\right)=t\left(\left[c_{1}^{-1} c_{1}^{\prime}\right], c_{2}\right)$ and extend it to the whole $\mathcal{A}\left(C_{2}\right)$ by linearity. The semigroup property $Q\left(c_{1}\right) Q\left(c_{2}\right)=Q\left(c_{1} c_{2}\right)$ follows automatically. The main point is to show that $Q\left(c_{1}\right)$ are shift invariant. We may trivially rewrite the characteristic property of the token as

$$
t\left(c_{1}^{\prime}, c_{2}^{\prime} c_{2}\right)=\int_{C_{1}} t\left(c_{1}, c_{2}^{\prime}\right) Q\left(c_{1}\right) t\left(c_{1}^{\prime}, c_{2}\right) d c_{1}
$$

which can be extended by linearity as $f\left(c_{2}^{\prime} c_{2}\right)=\int_{C_{1}} t\left(c_{1}, c_{2}^{\prime}\right) Q\left(c_{1}\right) f\left(c_{2}\right) d c_{1}$ to any function in $\mathcal{A}\left(C_{2}\right)$. Now replace $f$ by $Q\left(c_{1}^{\prime}\right) f$ to get

$$
Q\left(c_{1}^{\prime}\right) f\left(c_{2}^{\prime} c_{2}\right)=\int_{C_{1}} t\left(c_{1}, c_{2}^{\prime}\right) Q\left(c_{1}^{\prime} c_{1}\right) f\left(c_{2}\right) d c_{1} .
$$


But the left-hand side of the previous identity is nothing else as $\left[\lambda_{c_{2}^{\prime}} Q\left(c_{1}^{\prime}\right) f\right]\left(c_{2}\right)$ and the right-hand side is

$$
\begin{aligned}
\int_{C_{1}} t\left(c_{1}, c_{2}^{\prime}\right) Q\left(c_{1}^{\prime} c_{1}\right) f\left(c_{2}\right) d c_{1} & =Q\left(c_{1}^{\prime}\right)\left(\int_{C_{1}} t\left(c_{1}, c_{2}^{\prime}\right) Q\left(c_{1}\right) f\left(c_{2}\right) d c_{1}\right) \\
& =Q\left(c_{1}^{\prime}\right)\left(f\left(c_{2}^{\prime} c_{2}\right)\right) \\
& =\left[Q\left(c_{1}^{\prime}\right) \lambda_{c_{2}^{\prime}} f\right]\left(c_{2}\right),
\end{aligned}
$$

i.e. $Q$ is shift-invariant

The following Lemma is obvious.

Lemma 4.9. Let $q\left(c_{2}, c_{1}\right)$ be the kernel of $Q\left(c_{1}\right)$ as a convolution on $C_{2}$. Then the t-transform for the token $q\left(c_{2}, c_{1}\right)$ is a right inverse operator for the t-transform (3.19) with respect to $t\left(c_{1}, c_{2}\right)$.

The above results justify the following definition.

Definition 4.10. Let $t\left(c_{1}, c_{2}\right)$ be the basic distribution for a delta family $Q\left(c_{1}\right)$. Then we call $t\left(c_{1}, c_{2}\right)$, which is a token by Theorem 4.8, and the kernel $q\left(c_{2}, c_{1}\right)$ of $Q\left(c_{1}\right)$, which is a token by Proposition 4.4, dual tokens.

Example 4.11. The polynomials $t(n, x)=\frac{x^{n}}{n !}$ and distributions $q(n, x)=\delta^{(n)}(x)$ (which are kernels of derivative operators $D^{(k)}$ ) are canonical dual tokens.

As the reader may see in Theorem 4.8 we almost do not change proofs of [17: Section $2 /$ Theorem 1]. So we give our version of the next two results without proofs.

Theorem 4.12 (First Expansion Theorem). Let $S$ be a shift-invariant operator on $\mathcal{A}\left(C_{2}\right)$ and let $Q\left(c_{1}\right) \quad\left(c_{1} \in C_{1}\right)$ be a delta family with basic distribution $t\left(c_{1}, c_{2}\right)$. Then

$$
S=\int_{C_{1}} a\left(c_{1}\right) Q\left(c_{1}\right) d c_{1}
$$

with $a\left(c_{1}\right)=\left\langle s\left(c_{2}\right), t\left(c_{1}, c_{2}\right)\right\rangle_{c_{2}}=\left.S t\left(c_{1}, c_{2}\right)\right|_{c_{2}=e_{2}}$.

Theorem 4.13 (Isomorphism Theorem). Let $Q\left(c_{1}\right) \quad\left(c_{1} \in C_{1}\right)$ be a delta family with basic distribution $t\left(c_{1}, c_{2}\right)$. Then a mapping which carries a shift-invariant operator $S$ to a function

$$
s\left(c_{2}\right)=\int_{C_{1}} a\left(c_{1}\right) q\left(c_{2}, c_{1}\right) d c_{1}
$$

with $a\left(c_{1}\right)=\left\langle s\left(c_{2}\right), t\left(c_{1}, c_{2}\right)\right\rangle_{c_{2}}=\left.S t\left(c_{1}, c_{2}\right)\right|_{c_{2}=e_{2}}$ is an isomorphism of an operator algebra to a convolution algebra on $C_{2}$.

Example 4.14. For a sequence of binomial type $p_{n}$ and the associated delta operator $Q$ we can decompose a shift invariant operator $S$ on the space of polynomials as

$$
S=\sum_{k=0}^{\infty} a_{k} Q^{k}, \quad \text { where } a_{k}=\left[S p_{k}(x)\right]_{x=0}
$$


Example 4.15. Let a shift invariant operator $S$ on $\mathcal{H}_{2}\left(\mathbb{R}_{+}^{n+1}\right)$ be given by a kernel $s(v, t)$ :

$$
[S f](v, t)=\int_{\mathbb{R}_{+}^{n+1}} s\left(v^{\prime}, t^{\prime}\right) f\left(v+v^{\prime}, t+t^{\prime}\right) d v^{\prime} d t^{\prime} .
$$

Then we can represent $S$ by means of the delta family $Q(u) \quad\left(u \in \mathbb{R}^{n}\right)$ from Example 4.3 as

$$
S=\int_{\mathbb{R}^{n}} a(u) Q(u) d u \quad \text { where } a(u)=\int_{\mathbb{R}_{+}^{n+1}} s(v, t) P(u ; v, t) d v d t .
$$

And a similar representation is true for the Weierstrass kernel.

The following result has a somewhat cumbersome formulation and a completely evident proof. In the classic umbral calculus case the formulation turns to be very natural, but the proof is more hidden (see the next example).

Theorem 4.16. Let $t_{1}\left(c_{1}, c_{2}\right)$ and $t_{2}\left(c_{1}, c_{2}\right)$ be two tokens from $C_{1}$ to $C_{2}$, let the c-semigroup $C_{1}$ be equipped with a partial order relation $>$ and, moreover, let

$$
t_{2}\left(c_{1}, c_{2}\right)=\int_{C_{1}} a\left(c_{1}, c_{1}^{\prime}\right) t_{1}\left(c_{1}^{\prime}, c_{2}\right) d c_{1}^{\prime}
$$

where $a\left(c_{1}, c_{1}\right)=0$ whenever $c_{1}>c_{1}^{\prime}$. Let $Q_{2}\left(c_{1}\right)$ be a delta family associated to $t_{2}\left(c_{1}, c_{2}\right)$ and let $\sup _{t_{1}}(f) \in C_{1}$ denotes the support of $t$-transform of a function $f\left(c_{2}\right)$ with respect to $t_{1}\left(c_{1}, c_{2}\right)$. Then for any function $f\left(c_{2}\right)$ and $c_{1} \in C_{1}$

$$
\lambda_{c_{1}}\left(\sup _{t_{1}}\left(Q_{2}\left(c_{1}\right) f\right)\right) \subset \sup _{t_{1}}(f) .
$$

Proof. It is a direct consequence of the shift invariance of the delta family $Q_{2}\left(c_{1}\right)$ as operators on $C_{2}$ and Corollary 3.27

Example 4.17. The c-semigroup $\mathbb{N}_{+}$of natural numbers is naturally ordered. By the very definition [17: Section 3], for any polynomial sequence of binomial type, $p(k, x)$ is exactly of degree $k$ for all $k$. Thus for any two such sequences $p_{1}(k, x)$ and $p_{2}\left(k^{\prime}, x\right)$ we have

$$
p_{1}(k, x)=\sum_{k^{\prime}=1}^{k} a\left(k, k^{\prime}\right) p_{2}\left(k^{\prime}, x\right),
$$

i.e. (4.1) is satisfied. Let now $Q_{2}(k)=Q^{k}$ be the delta family associated to $p_{2}(k, x)$. Then by Theorem 4.16 the polynomial $Q_{2}(k) p_{1}\left(k^{\prime}, x\right)$ if of degree $k^{\prime}-k$. This gives [17: Section 2/Propositions 1 and 2].

Generally speaking, an order on a set of combinatorial numbers allows to consider some recurrence relations, which express a combinatorial number via smaller numbers of the same kind. We will return to this subject in Subsection 4.3 in connection with generating functions.

4.2 Generating functions and umbral functionals. It is known that generating functions are a powerful tool in combinatorics. We try to interpret the notion of generating functions in our terms. Particularly, we will treat [7: Examples 4.5 - 4.9] accordingly. 
Definition 4.18. Let the function $f\left(c_{1}\right)$ represents a set of combinatorial quantities indexed by points of a c-semigroup $C_{1}$ (a combinatorial function for short). Let $C_{2}$ be another c-semigroup and $t\left(c_{1}, c_{2}\right)$ be a token between them. The t-transform

$$
\hat{f}\left(c_{2}\right)=\int_{C_{1}} f\left(c_{1}\right) t\left(c_{1}, c_{2}\right) d c_{1}
$$

of $f\left(c_{1}\right)$ is called generating function for $f\left(c_{1}\right)$ (with respect to $C_{2}$ and $t\left(c_{1}, c_{2}\right)$ ).

Following [14] an exceedingly useful method for defining a function $f\left(c_{1}\right)$ is to apply an umbral linear functional $l$ to a token $t\left(c_{1}, c_{2}\right)$ :

$$
f\left(c_{1}\right)=\left\langle l, t\left(c_{1}, c_{2}\right)\right\rangle:=\int_{C_{2}} l\left(c_{2}\right) t\left(c_{1}, c_{2}\right) d c_{2} .
$$

If we now consider the generating function $\hat{f}\left(c_{2}\right)$ with respect to the token $q\left(c_{2}, c_{1}\right)$, which is dual to $t\left(c_{1}, c_{2}\right)$, then we will found that

$$
\begin{aligned}
\hat{f}\left(c_{2}\right) & =\int_{C_{1}} f\left(c_{1}\right) q\left(c_{2}, c_{1}\right) d c_{1} \\
& =\int_{C_{1}} \int_{C_{2}} l\left(c_{2}^{\prime}\right) t\left(c_{1}, c_{2}^{\prime}\right) d c_{2}^{\prime} q\left(c_{2}, c_{1}\right) d c_{1} \\
& =\int_{C_{2}} l\left(c_{2}^{\prime}\right) \int_{C_{1}} t\left(c_{1}, c_{2}^{\prime}\right) q\left(c_{2}, c_{1}\right) d c_{1} d c_{2}^{\prime} \\
& =\int_{C_{2}} l\left(c_{2}^{\prime}\right) \delta\left(c_{2}^{\prime}, c_{2}\right) d c_{2}^{\prime} \\
& =l\left(c_{2}\right) .
\end{aligned}
$$

So we found the following simple connection between umbral functionals and generating functions:

Theorem 4.19. The generating function $\hat{f}\left(c_{2}\right)$ for a combinatorial function $f\left(c_{1}\right)$ with respect to a token $q\left(c_{2}, c_{1}\right)$ is the kernel of the umbral functional for $f\left(c_{1}\right)$ with respect to the token $p\left(c_{1}, c_{2}\right)$ dual to token $q\left(c_{2}, c_{1}\right)$.

Example 4.20. Let $\left\{f_{n}\right\}_{n=0}^{\infty}$ be the sequence of Fibonacci numbers and let $F$ be the generating function associated to $\left\{f_{n}\right\}_{n=0}^{\infty}$ by the token $q(k, x)=\delta^{(k)}(x)$ :

$$
\begin{aligned}
F(x) & =\sum_{k=0}^{\infty} f_{n} \delta^{(k)}(x) \quad\left[=\frac{1}{2 \pi} \int_{-\infty}^{\infty} \frac{1}{1-(i \xi)-(i \xi)^{2}} e^{-i \xi x} d \xi\right] \\
& =\frac{1}{\sqrt{5}} e^{\frac{1-\sqrt{5}}{2} x}\left(1+\left(e^{\sqrt{5} x}-1\right) \chi(-x)\right)
\end{aligned}
$$

where $\chi$ is the Heaviside function, i.e. $\chi(x)=\left\{\begin{array}{ll}0 & \text { if } x<0 \\ 1 & \text { if } x \geq 0 .\end{array}\right.$ Then $F$ is the kernel of an umbral functional, which generates the sequence $\left\{f_{n}\right\}_{n=0}^{\infty}$ from the polynomial sequence 
$t(n, x)=\frac{x^{n}}{n !}$ which is dual to $q(k, x)=\delta^{(k)}(x)$ (Example 4.11): $f_{n}=\int_{-\infty}^{\infty} F(x) \frac{x^{n}}{n !} d x$. In this way we obtain a constructive solution for the classic moment problem [1].

4.3 Recurrence operators and generating functions. We are looking for reasons why some generating functions are better suited to handle given combinatorial functions than others. The answer is given by their connections with recurrence operators.

We will speak on combinatorial functions here that are functions defined on csemigroups. Different numbers (Bell, Fibonacci, Stirling, etc.) known in combinatorics are, of course, combinatorial functions defined on the c-semigroup $\mathbb{N}_{+}$.

Definition 4.21. An operator $R$ on $C_{1}$ is said to be a recurrence operator with respect to a token $t\left(c_{1}, c_{2}\right)$ for a combinatorial function $f\left(c_{1}\right)$ if

$$
[R f]\left(c_{1}\right)=t\left(c_{1}, e_{2}\right) .
$$

Particularly, $[R f]\left(c_{1}\right)$ has a reproducing property from Lemma 3.19.

If we consider an algebra of convolutions of combinatorial functions, then by the general property of t-transforms it is isomorphic to convolution algebra of their generating function, which occasionally can be isomorphic to an algebra with multiplication (multiplication of formal power series, exponential power series, Dirichlet series, etc.). This was already observed by other means in [7].

The following theorem connects generating functions and recurrent operators.

Theorem 4.22. Let $C_{1}, C_{2}$ be c-semigroups with a token $t\left(c_{1}, c_{2}\right)$ between them, let $q\left(c_{2}, c_{1}\right)$ be its dual token and $f\left(c_{1}\right)$ a combinatorial function with corresponding recurrence operator $R$ defined by its kernel $r\left(c_{1}, c_{1}^{\prime}\right)$ as $[R f]\left(c_{1}\right)=\int_{C_{1}} r\left(c_{1}, c_{1}^{\prime}\right) f\left(c_{1}^{\prime}\right) d c_{1}^{\prime}$. Then the generating function $\hat{f}\left(c_{1}\right)$ satisfies the equation

$$
[\tilde{R} \hat{f}]\left(c_{2}\right)=t\left(e_{1}, c_{2}\right)
$$

where $\tilde{R}$ is defined by the kernel

$$
\tilde{r}\left(c_{2}, c_{2}^{\prime}\right)=\int_{C_{1}} \int_{C_{1}} t\left(c_{1}^{\prime \prime}, c_{2}\right) r\left(c_{1}^{\prime \prime}, c_{1}^{\prime}\right) q\left(c_{1}^{\prime}, c_{2}^{\prime}\right) d c_{1}^{\prime} d c_{1}^{\prime \prime} .
$$

Proof. We start from the observation that

$$
\int_{C_{2}} \tilde{r}\left(c_{2}, c_{2}^{\prime}\right) t\left(c_{1}, c_{2}^{\prime}\right) d c_{2}^{\prime}=\int_{C_{1}} t\left(c_{1}^{\prime \prime}, c_{2}\right) r\left(c_{1}^{\prime \prime}, c_{1}\right) d c_{1}^{\prime \prime}
$$

which follows from application to both sides of (4.5) the integral operator with kernel $t\left(c_{1}, c_{2}^{\prime}\right)$ and the identity $\int_{C_{2}} q\left(c_{1}^{\prime}, c_{2}^{\prime}\right) t\left(c_{2}^{\prime}, c_{1}\right) d c_{2}^{\prime}=\delta\left(c_{1}^{\prime}, c_{1}\right)$. Then

$$
\begin{aligned}
{[\tilde{R} \hat{f}]\left(c_{2}\right) } & =\int_{C_{2}} \tilde{r}\left(c_{2}, c_{2}^{\prime}\right) \hat{f}\left(c_{2}^{\prime}\right) d c_{2} \\
& =\int_{C_{2}} \tilde{r}\left(c_{2}, c_{2}^{\prime}\right) \int_{C_{1}} f\left(c_{1}\right) t\left(c_{1}, c_{2}^{\prime}\right) d c_{1} d c_{2} \\
& =\int_{C_{1}} \int_{C_{2}} \tilde{r}\left(c_{2}, c_{2}^{\prime}\right) t\left(c_{1}, c_{2}^{\prime}\right) d c_{2} f\left(c_{1}\right) d c_{1}
\end{aligned}
$$


and, further on,

$$
\begin{aligned}
{[\tilde{R} \hat{f}]\left(c_{2}\right) } & =\int_{C_{1}} \int_{C_{1}} t\left(c_{1}^{\prime \prime}, c_{2}\right) r\left(c_{1}^{\prime \prime}, c_{1}\right) d c_{1}^{\prime \prime} f\left(c_{1}\right) d c_{1} \\
& =\int_{C_{1}} t\left(c_{1}^{\prime \prime}, c_{2}\right) \int_{C_{1}} r\left(c_{1}^{\prime \prime}, c_{1}\right) f\left(c_{1}\right) d c_{1} d c_{1}^{\prime \prime} \\
& =\int_{C_{1}} t\left(c_{1}^{\prime \prime}, c_{2}\right) \delta\left(c_{1}^{\prime \prime}\right) d c_{1}^{\prime \prime} \\
& =t\left(e_{1}, c_{2}\right)
\end{aligned}
$$

where we deduce (4.7) from (4.6)

In fact, our calculation just says that $\tilde{R} g=T \delta$ for $g=T f$ and operator $\tilde{R}=T R T^{-1}$ if $R f=\delta$. A touch of non-triviality appears when we use special properties of functions $t\left(c_{1}, c_{2}\right)$ and $q\left(c_{2}, c_{1}\right)$ be a pair of dual tokens:

Corollary 4.23. If under the assumptions of Theorem 4.22 the operator $R$ is shiftinvariant on $C_{1}$, i.e. has a kernel of the form $r\left(c_{1}, c_{1}^{\prime}\right)=r\left(c_{1}^{\prime-1} c_{1}\right)$, then the operator $\tilde{R}$ is also shift-invariant on $C_{2}$ with a kernel of the form $\tilde{r}\left(c_{2}, c_{2}^{\prime}\right)=r\left(c_{2}^{\prime-1} c_{2}\right)$.

Proof. It follows from the property of t-transforms to map a shift-invariant operator on $C_{1}$ to a shift-invariant operator on $C_{2}$

Remark 4.24. The above corollary clearly indicates what type of generating functions is reasonable: a simple generating function can be constructed with respect to a token $t\left(c_{1}, c_{2}\right)$ such that the recurrence operator $R$ is shift-invariant with respect to it.

As it usually occurs, a simple fact may have interesting realizations.

Example 4.25. Let $f_{n}$ be the Fibonacci numbers. Their known recursion $f_{n}=$ $f_{n-1}+f_{n-2}$ should be stated in a more accurate way with the help of agreement (3.5) as $f_{n}-f_{n-1}-f_{n-2}=\delta_{n, 0} \quad(n \geq 0)$. Then the recurrence operator is given by $R=$ $I-S-S^{2}$, where $S$ is a backward shift $S f(n)=f(n-1)$ on $\mathbb{N}_{+}$, particularly, $R$ is shift-invariant with respect to the token $t(x, n)=x^{n}$. The corresponding kernel is $r(n, i)=\delta_{n, i}-\delta_{n-1, i}-\delta_{n-2, i}$ which is a function of $n-i$. For the pair of dual tokens $t(x, n)=x^{n}$ and $q(i, y)=\frac{\delta^{(i)}(y)}{i !}$ we obtain a transformed kernel:

$$
\begin{aligned}
\tilde{r}(x, y) & =\sum_{n=0}^{\infty} \sum_{i=0}^{\infty} t(x, n) r(n, i) q(i, y) \\
& =\sum_{n=0}^{\infty} \sum_{i=0}^{\infty} x^{n}\left(\delta_{n, i}-\delta_{n-1, i}-\delta_{n-2, i}\right) \frac{\delta^{(i)}(y)}{i !} \\
& =\sum_{n=0}^{\infty} x^{n}\left(1-x-x^{2}\right) \frac{\delta^{(n)}(y)}{n !} \\
& =\left(1-x-x^{2}\right) \sum_{n=0}^{\infty} x^{n} \frac{\delta^{(n)}(y)}{n !} .
\end{aligned}
$$


From the Taylor expansion the sum in (4.8) obviously represents an integral kernel of the identity operator, therefore the transformation $\tilde{R}$ is the operator of multiplication by $1-x-x^{2}$ and the generating function $\hat{f}$ should satisfy $\tilde{R} f(x)=\left(1-x-x^{2}\right) \hat{f}(x)=x^{0}=1$. Thus

$$
\hat{f}(x)=\sum_{n=0}^{\infty} f_{n} x^{n}=\frac{1}{1-x-x^{2}} .
$$

Note that the operator $\tilde{R}$ of multiplication by $\left(1-x-x^{2}\right)$ is "shift-invariant" - it commutes with the operator of multiplication by $x$ (both these operators are certain convolutions under Fourier transform).

An attempt to construct a transformation $\tilde{R}$ with respect to a similar pair of dual tokens $t_{1}(x, n)=\frac{x^{n}}{n !}$ and $q_{1}(i, y)=\delta^{(i)}(y)$ will not enjoy the above simplicity.

Example 4.26. Let $B_{n}$ be the Bell numbers (see [14] or [18: Section 3]). The known recursion for them [18: Section 3] can again be restated as

$$
B_{n}-\sum_{k=0}^{n-1}\left(\begin{array}{c}
n-1 \\
k
\end{array}\right) B_{k}=\delta_{n, 0} \quad(n \geq 0) .
$$

Such a recurrence identity is shift-invariant with respect to $W$ defined by the identity $W f(n)=\frac{f(n-1)}{n}$ on $\mathbb{N}_{+}$. In other words, it is shift-invariant with respect to the token $t(x, n)=\frac{x^{n}}{n !}$. The kernel $r(n, i)$ corresponding to the recurrence operator $R$ in (4.9) is

$$
r(n, i)=\delta_{n, i}-\sum_{k=0}^{n-1}\left(\begin{array}{l}
n \\
k
\end{array}\right) \delta_{k, i} .
$$

We apply a transformation with respect to a pair of dual tokens $t(x, n)=\frac{x^{n}}{n !}$ and $q(i, y)=\delta^{(i)}(y)$ by

$$
\begin{aligned}
\tilde{r}(x, y) & =\sum_{n=0}^{\infty} \sum_{i=0}^{\infty} t(x, n) r(n, i) q(i, y) \\
& =\sum_{n=0}^{\infty} \sum_{i=0}^{\infty} \frac{x^{n}}{n !}\left(\delta_{n, i}-\sum_{k=0}^{n-1}\left(\begin{array}{c}
n-1 \\
k
\end{array}\right) \delta_{k, i}\right) \delta^{(i)}(y) \\
& =\sum_{n=0}^{\infty} \sum_{i=0}^{\infty} \frac{x^{n}}{n !} \delta_{n, i} \delta^{(i)}(y)-\sum_{n=0}^{\infty} \sum_{i=0}^{\infty} \frac{x^{n}}{n !} \sum_{k=0}^{n-1}\left(\begin{array}{c}
n-1 \\
k
\end{array}\right) \delta_{k, i} \delta^{(i)}(y) \\
& =\sum_{n=0}^{\infty} \frac{x^{n}}{n !} \delta^{(n)}(y)-\sum_{n=0}^{\infty} \sum_{i=0}^{n-1} \frac{x^{n}}{n !} \frac{(n-1) !}{i !(n-1-i) !} \delta^{(i)}(y) \\
& =\sum_{n=0}^{\infty} x^{n} \frac{\delta^{(n)}(y)}{n !}-\sum_{n=0}^{\infty} \sum_{i=0}^{n-1} \frac{1}{n} x^{n} \frac{\delta^{(i)}(y)}{i !(n-1-i) !} \\
& =\sum_{n=0}^{\infty} x^{n} \frac{\delta^{(n)}(y)}{n !}-\sum_{n=0}^{\infty} \frac{x}{n} \sum_{i=0}^{n-1} \frac{x^{n-i-1}}{(n-1-i) !} \frac{x^{i} \delta^{(i)}(y)}{i !}
\end{aligned}
$$


One verifies that integration of a function $f(y)$ with $\operatorname{sum} \sum_{i=0}^{n-1} \frac{x^{n-i-1}}{(n-1-i) !} \frac{x^{i} \delta^{(i)}(y)}{i !}$ in (4.10) produces the $(n-1)$-th term in the Taylor expansion of the product $e^{x} f(x)$. Thus the entire expression in (4.10) is a kernel of the operator: $\tilde{R}=I-\int e^{x}$ where $I$ is the identity operator and $\int$ is the operator of the anti-derivation fixed by the condition $\left[\int g\right](0)=0$, particularly $\int \frac{x^{n}}{n !}=\frac{x^{n+1}}{(n+1) !}$. So the generating function $\hat{b}$ should satisfy the equation $\left(I-\int e^{x}\right) \hat{b}(x)=x^{0}=1$. Taking the derivative from both sides we found the differential equation $\left(D-e^{x}\right) \hat{b}(x)=0$. With the obvious initial condition $\hat{b}(0)=1$ it determines that $\hat{b}(x)=\exp (\exp (x)-1)$.

One may interpret (4.3) as if $f\left(c_{1}\right)$ is the fundamental solution to operator $R$. This is indeed a right way of thinking if one can found a group action such that $R$ is shiftinvariant. Then the convolution $h\left(c_{1}\right)=[g * f]\left(c_{1}\right)$ will give a solution to the equation $[R h]\left(c_{1}\right)=g\left(c_{1}\right)$.

\section{Models for the umbral Calculus}

We present realizations (models) for different descriptions of the umbral calculus mentioned in Section 2.

5.1 Finite operator description: a realization. As was mentioned in Subsection 2.1, the main ingredients of the approach are polynomial sequences of binomial type and shift invariant operators. As was already pointed in Examples 3.15 and 3.21, the notion of token is a useful refinement of polynomial sequences of binomial type. It is much more useful if we consider it together with shift invariant operators on c-semigroups not just groups. The next subsections contains some details of this.

5.2 Hopf algebra description: a Realization. We describe some applications of c-semigroups and tokens. First we can add new lines to Proposition 3.11:

Proposition 5.1. Let $C_{2}$ be a c-semigroup with a right source $e$, let $C_{1}$ be another c-semigroup and $t\left(c_{1}, c_{2}\right)$ be a token between them. Then the list in Proposition 3.11 for $C_{2}$ can be extended via t-transforms by

4. the subspace of convolutions over $C_{1}$

5. the subspace of linear functionals over $\mathcal{C}\left(C_{1}\right)$.

Proof. By Theorem 3.26 the space of convolutions over $C_{2}$ can be mapped to the space of convolutions over $C_{1}$ via a t-transform. A backward mapping from convolutions over $C_{1}$ to convolutions over $C_{2}$ is given by the dual token $q\left(c_{2}, c_{1}\right)$ of the associated delta family $Q\left(c_{1}\right)$

We establish the correspondence of four mentioned sets as linear spaces. However, two of them (convolutions over $C_{1}$ and $C_{2}$ ) have isomorphic structures as convolution algebras. This allows us to transfer also their multiplication law on the space of linear functionals. 
Definition 5.2. The product $l=l_{1} * l_{2}$ of two functionals $l_{1}$ and $l_{2}$ is again a linear functional corresponding to the convolution $S=S_{1} * S_{2}$ where the convolutions $S_{1}$ and $S_{2}$ correspond to $l_{1}$ and $l_{2}$. It acts on a function $p\left(c_{1}\right)$ as

$$
\left\langle l, p\left(c_{1}^{\prime}, \cdot\right)\right\rangle=\int_{C_{1}}\left\langle l_{1}, p\left(c_{1}, \cdot\right)\right\rangle\left\langle l_{2}, p\left(\left[c_{1}^{-1} c_{1}^{\prime}\right], \cdot\right)\right\rangle d c_{1} .
$$

Example 5.3. We return to the notations of Examples 3.15 and 3.21. For a linear functional $l$ on $C_{2}=\mathbb{R}$ we introduce its t-transform $[\mathcal{T} l](n)$ as its action (with respect to the $x$ variable) on the token $t(n, x)=p_{n}(x)$ given by a sequence of polynomial type $p_{n}:[\mathcal{T} l](n)=l T(n, x)=l p_{n}(x)$. Then the product $l$ of two functionals $l_{1}$ and $l_{2}$ are defined by the convolution on $\mathbb{N}_{+}$:

$$
[\mathcal{T} l](n)=\int_{\mathbb{N}_{+}} l_{1}(k) l_{2}\left(\left[k^{-1} n\right]\right) d n=\sum_{k=0}^{n} l_{1}(k) l_{2}(n-k) .
$$

The above definition of a product can be found in [16]. The c-semigroup algebra over $\mathbb{N}_{+}$can be naturally realized as the multiplicative algebra of a formal power series of one variable $t$ (see Proposition 3.6). So we obtain one more face an umbral algebras as follows:

Corollary 5.4 (see [16]). The algebra of linear functionals on $\mathbb{R}$ with Hopf multiplication (5.1) is in a natural correspondence $\left\{l_{n}\right\}_{n=0}^{\infty} \mapsto \sum_{n=o}^{\infty} l_{n} t^{n}$ with the algebra of formal power series in one variable $t$.

5.3 The semantic description: a realization. We start from the c-semigroup $S$, which is the direct product of many copies of the c-semigroup $\mathbb{N}_{+}$. Different copies of $\mathbb{N}_{+}$ are labelled by letters of an alphabet $A$, which is denoted by Greek letters. An element $k$ of a copy $\mathbb{N}_{+}$labelled by a letter $\alpha$ can be written interchangeably as $k_{\alpha}$ or $\alpha^{k}$. In this sense any function $p\left(k_{\alpha}, l_{\beta}, \ldots\right)$ in $\mathcal{C}_{0}(S)$ can be identified with the polynomial

$$
\mathrm{p}(\alpha, \beta, \ldots)=\sum_{k_{\alpha}, l_{\beta}, \ldots} p\left(k_{\alpha}, l_{\beta}, \ldots\right) \alpha^{k} \beta^{l} \ldots .
$$

More over this identification send the convolution $p * q$ of two functions $p, q \in \mathcal{C}_{0}(S)$ over $S$ to the product of polynomials pq. So we will not distinguish a function $p\left(k_{\alpha}, l_{\beta}, \ldots\right)$ and the corresponding polynomial $\mathrm{p}(\alpha, \beta, \ldots)$ any more as well as the convolution algebra $\mathcal{C}_{0}(S)$ and polynomial ring $D[A]$ over the alphabet $A$.

A linear functional eval is defined as

$$
\operatorname{eval}(p)=\int_{S} p\left(k_{\alpha}, l_{\beta}, \ldots\right) d s
$$

via a measure $d s$ on $S$ such that $d s\left(1_{\alpha}\right)=1$ for any $\alpha \in A$. Because every $p$ has compact (i.e. finite) support in $S$ the integration does not generate any difficulties. Equality (2.4) expresses the fact that $S$ is the direct sum of $\mathbb{N}_{+}$'s and the measure $d s$ is the direct product of corresponding measures $d s_{\alpha}$. 
In spirit of 2.1.4 we require that the measure $d s_{\varepsilon}$ on the copy $\mathbb{N}_{+}$labelled by the distinguished umbra $\varepsilon$ is defined by the sequence $(1,0,0,0, \ldots)$.

Now eval defines an equivalence relation - umbral equivalence on $\mathcal{C}_{0}(S)$, namely $f \simeq g \quad\left(f, g \in \mathcal{C}_{0}(S)\right)$ if $(f-g)$ belongs to the kernel of eval (i.e. $\operatorname{eval}(f-g)=$ $0)$. Because eval is additive but not multiplicative we see that umbral equivalence is invariant under addition but multiplication (unlike the support of functions belongs to different components in the direct product of $\mathbb{N}_{+}$).

Finally, we will define $n \cdot \beta$ as a function

$$
\beta^{1} \otimes \beta^{0} \otimes \cdots \otimes \beta^{0}+\beta^{0} \otimes \beta^{1} \otimes \cdots \otimes \beta^{0}+\beta^{0} \otimes \beta^{0} \otimes \cdots \otimes \beta^{1}
$$

in the $n$-th tensor power $\otimes_{k=1}^{n} \mathbb{N}_{+}(\beta)$ of a copy of $\mathbb{N}_{+}$labelled by $\beta$ (this means that eval has identical distribution on all of them).

This gives a realization for classical umbral calculus described via a semantic approach in $[18,19]$.

Acknowledgments. This paper was inspired by works of Gian-Carlo Rota and his personal encouragement and interest supported me during its preparation. V. Kharchenko kindly enlightened me in the field of algebra. I am grateful to anonymous referees who took a hard job to read the initial version of the paper and suggested innumerable improvements and corrections.

The essential part of the paper was written while author's stay at the Department of Mathematical Analysis, University of Ghent, as a Visiting Postdoctoral Fellow of the FWO-Vlaanderen (Fund of Scientific Research- Flanders), Scientific Research Network 3GP03196 "Fundamental Methods and Technique in Mathematics", whose hospitality h gratefully acknowledges.

\section{References}

[1] Akhiezer, N. I.: Classical Moment Problem (in Russian). Moscow: Gos. Izd. Fis.-Mat. Lit. 1961; Engl. transl.: New York: Hafner Publ. Co. 1965.

[2] Akhiezer, N. I.: Lectures on Integral Transforms. Providence (RI): Amer. Math. Soc. 1988.

[3] Artamonov, V. A. et al.: Genereal Algebra, Vol. 2 (in Russian). Moscow: Nauka 1991.

[4] Barnabei, M., Brini, A. and G. Nicoletti: Polynomial sequences of integral type. J. Math. Anal. Appl. 78 (1980), $598-617$.

[5] Barnabei, M., Brini, A. and G.-C. Rota: Section coefficients and section sequences. Atti Accad. Naz. Lincei Rend., Cl. Sci. Fis. Mat. Natur. (8) 68 (1980)1, 5 - 12 (reprinted in [11: pp. $337-344])$.

[6] Connes, A.: Non-Commutative Geometry. New York: Acad. Press 1994.

[7] Doubilet, P., Rota, G.-C. and R. Stanley: On the foundation of combinatorial theory. Part VI: The idea of generating function. In: Proceedings of the Sixth Berkelay Symposium on Mathematical Statistics and Probability, Volume 2: Probability Theory ( eds.: L.M. Le Cam, etc.). Berkeley: Univ. California Press 1971, pp. $267-318$ (reprinted in [15: pp. 83 - 134] and [11: pp. 148 - 199]). 
[8] Evans, L. C.: Partial Differential Equations. Providence (RI): Amer. Math. Soc. 1998.

[9] Kirillov, A. A. and A. D. Gvishiani: Theorems and Problems in Functional Analysis (Problem Books in Mathematics). New York: Springer-Verlag 1982.

[10] Knuth, D. K.: Convolution polynomials. Preprint. Stanford: University 1996. http://www-cs-faculty.stanford.edu/ knuth/papers/cp.tex.gz.

[11] Kung, J. P. S. (ed.): Gian-Carlo Rota on Combinatorics: Introductory Papers and Commentaries. (Contemporary Mathematicians: Vol. 1). Boston: Birkhäuser Verlag 1995.

[12] Mullin, R. and G.-C. Rota: On the foundation of combinatorial theory. Part III: Theory of binomial enumeration. In: Graph Theory and Its Applications (ed.: B. Harris). New York: Acad. Press 1970, pp. $167-213$ (reprinted in [11: pp. $118-147]$ ).

[13] Roman, S. and G.-C. Rota: The umbral calculus. Adv. Math. 27 (1978), 95 - 188.

[14] Rota, G.-C.: The number of partitions of a set. Amer. Math. Monthly 71 (1964), 498 504 (reprinted in [15: pp. $1-6]$ and [11: pp. $111-117]$ ).

[15] Rota, G.-C.: Finite Operator Calculus. New York: Acad. Press 1975.

[16] Rota, G.-C.: Hopf algebras methods in combinatorics. In: Problèmes Combinatoires et Théorie des Graphes (Coll. Intern. du Centre Nat. de la Rech. Sci.: Vol. 260). Paris: Centre National de la Recherche Scientifique 1978, pp. 363 - 365 (reprinted in [11: pp. $287-289])$.

[17] Rota, G.-C., Kahaner, D. and A. Odlyzko: Finite operator calculus. J. Math. Anal. Appl. 42 (1973), 685 - 760 (reprinted in [15: pp. $7-82]$ ).

[18] Rota, G.-C. and B. D. Taylor: An introduction to the umbral calculus. In: Analysis, Geometry and Groups: A Riemann Legacy Volume (eds.: H. M. Srivastava and Th. M. Rassias). Palm Harbor (Florida): Hadronic Press 1983, pp. 513 - 525.

[19] Rota, G.-C. and B. D. Taylor: The classical umbral calculus. SIAM J. Math. Anal. 25 (1994), $694-711$.

[20] Shabat, B. V.: Introduction into Komplex Analysis, Vol. I: Functions of a Single Variable, 3rd ed. Moscow: Nauka 1985.

[21] Stanley, R. P.: Foundation I and the development of algebraic combinatorics. In [11: pp. $105-107]$.

[22] Weinstein, A.: Groupoids: Unifying internal and external symmetries. Notices Amer. Math. Soc. 43 (1996), $744-752$.

Received 23.01.1998 ${ }^{2)}$; in revised form 11.02.2000

2) See also http://arXiv.org/abs/funct-an/9704001/ 